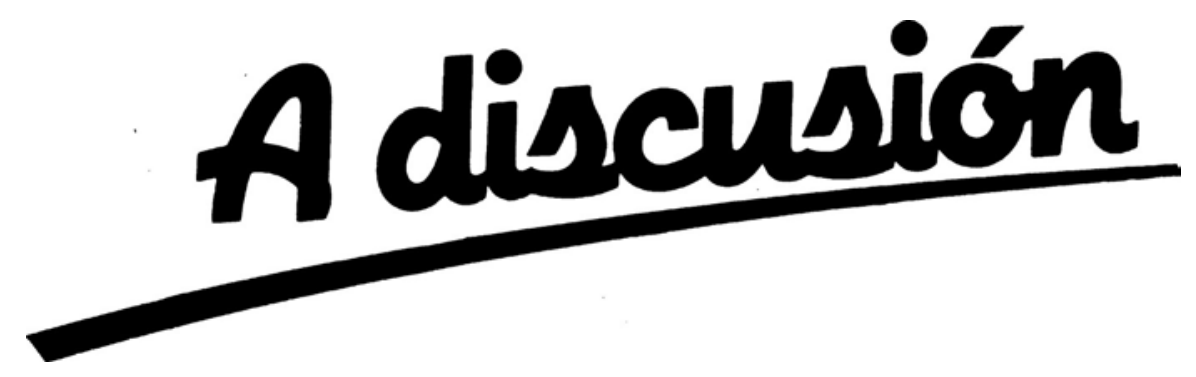

TWO-STAGE HUBER ESTIMATION*

Tae-Hwan Kim and Christophe Muller**

WP-AD 2005-17

Corresponding author: C. Muller. Universidad de Alicante. Departamento de Fundamentos del Análisis Económico, Campus de San Vicente, 03080 Alicante (Spain). Tel.: +34965903400 / Fax: +34965903898 / E-mail: cmuller@merlin.fae.ua.es.

Editor: Instituto Valenciano de Investigaciones Económicas, S.A.

Primera Edición Abril 2005

Depósito Legal: V-2050-2005

IVIE working papers offer in advance the results of economic research under way in order to encourage a discussion process before sending them to scientific journals for their final publication.

\footnotetext{
* This research has been supported under the British Academy Award No. 30,649. The second author is also grateful for the financial support by Spanish Ministry of Sciences and Technology. Project No. BEC2002-03097 and by the Instituto Valenciano de Investigaciones Economicas. We thank two referees for their useful comments. Usual disclaimers apply.
}

** T-H. Kim: Yonsei University. C. Muller: Universidad de Alicante. 


\section{TWO-STAGE HUBER ESTIMATION}

\section{Tae-Hwan Kim and Christophe Muller}

\section{A B S T R A C T}

In this paper we study how the Huber estimator can be adapted to the presence of endogeneity in a two stage equations setting similar to that of 2 SLS. We propose an estimation procedure that is at the same time relatively (i) simple, (ii) robust and (iii) efficient. Moreover, we deal with the case of random regressors and asymmetric errors, two extensions rarely present in this literature. The preliminary scale correction is implemented with median absolute deviation estimator, which is consistent with our above criteria and is a very robust estimator of scale. The resulting estimator is termed as the Two-Stage Huber (2SH) estimator.

We explicitly establish the conditions for consistency and asymptotic normality of the $2 \mathrm{SH}$ estimator and we derive the formula of the asymptotic covariance matrix. We conduct Monte Carlo simulations whose results indicate that the 2SH estimator has smaller standard errors than the Two-Stage Least Squares (2SLS) estimator and than the Two-Stage Least Absolute Deviations (2SLAD) estimator in many situations. On the whole, the 2SH estimator appears to be a simple and useful alternative to 2SLS and 2SLAD in cases of two-stage estimation to deal with endogeneity when there are concerns for both robustness and efficiency.

Keywords: Two-stage estimation, Huber estimation, robustness, endogeneity. 


\section{Introduction}

The endogeneity problem in model estimation is usually dealt with by conducting the estimation in two stages. In the first stage, reduced form equations are estimated and the fitted values of endogenous variables are calculated. Then, these fitted values are used as regressors in the second stage and the covariance matrix of the estimated parameters is corrected for the replacement of the initial regressors by their fitted values. The use of twostage least-squares (2SLS) to treat the endogeneity problem in a linear model is an example of this approach. Estimation methods in two stages have been studied for many M-estimators. For example, see Malinvaud (1970), Heckman (1978), Amemiya(1985), Krasker and Welsch (1985), Newey (1985, 89, 94), Krasker (1986), Pagan (1986), Duncan (1987). In this paper, we extend the class of two-stage robust estimators in the context of a linear regression model under endogeneity of some explanatory variables.

The motivation of this research is to provide an estimation procedure that is at the same time relatively (i) simple to implement, (ii) robust, (iii) efficient. Moreover, we want this estimation procedure to be able to deal easily with the cases of asymmetric errors and random regressors arising in two-stage setting of equations familiar to empirical researchers who focus their interest on the 'structural equation'. This seems a reasonable requirement for a method to be used in applied work in many situations.

The preliminary scale correction is implemented with median absolute deviation estimator, which is consistent with our above criteria and is sometimes considered as the 'most robust estimator of scale'.

We are interested in the case in which the two stages are implemented by using the Huber estimator (Huber, 1964, 1981), one of the main robust estimators, which guarantees the robustness of the procedure with respect to error terms. We denote this procedure Two-Stage Huber (2SH) Estimator. Typically, robust estimators attempt to respond to a variety of problems: outliers generating heavy tails errors in the dependent variable; true distributions of errors deviating from the assumed distribution (generally Gaussian distribution); other model misspecifications ${ }^{1}$. Using the same Huber estimator in the first stage not only makes the procedure simple to implement, but also eliminates the potential influence of non-normality or outliers in the first stage.

A few authors have tackled the problem of robust estimation for simultaneous equation systems. Krasker and Welsch (1985), Krasker (1986), Koenker and Portnoy (1990), Krishnakumar and Ronchetti (1997), Maronna and Yohai (1997), Flavin (1999) propose estimators based on weighted instrumental variables or on bounded influence regressions. Krasker and Welsch propose weighted IV resistant estimators defined by implicit equa-

\footnotetext{
${ }^{1}$ See for example Huber $(1964,1966)$, Krasker and Welsch (1985).
} 
tions. These estimators have attractive robust properties but can only be calculated through an iterative procedure. Koenker and Portnoy (1990) study weighted LAD estimators that improve efficiency as compared to simple LAD estimators. Krishnakumar and Ronchetti propose B-robust (i.e. with bounded influence function) estimators as a good compromise between efficiency and robustness. Their estimators are based on the application of the Huber function to a residual that is an affine combination of the score of the considered system when errors are normal. As for Krasker and Welsch estimator, the calculus of the estimator is not simple in that it requires an iterative procedure. In an empirical paper, Flavin (1999) robustifies the first-order conditions of an IV estimator, and uses the Median Absolute Deviation (MAD) as a scale estimator. Maronna and Yohai (1997) review some of these methods and identify three different estimating strategies; robustifying three-stage least squares, robustifying the full information maximum likelihood method and generalizing multivariate $\tau$-estimators.

Rather than following these strategies, we focus on a natural extension of the Huber estimator to the two-stage setting used in most applied empirical work. Indeed, the two-stage setting is the one likely to be used by applied researchers who are predominantly interested in the second stage estimates. Moreover, the successive Huber estimation is simple to implement, as opposed to many other robust estimators. Krasker (1986) proposes to robustify the two-stage least-square method by replacing each OLS stage by a bounded influence estimator and to apply it to simultaneous equations. We follow this approach by replacing each OLS stage with a modified Huber estimator for which we use the mean absolute deviation estimator (MAD) as preliminary rescaling estimator for the errors at each estimation stage. This feature modifying the classical Huber estimator has the advantage of being simple and quick to apply, and therefore to allow estimation procedures only based on usual Huber, Least-Squares and Least-Absolute Deviation estimators, all for which readily usable commands are available in many statistical packages (e.g. Stata). In a sense, our procedure is akin to that of Krishnakumar and Ronchetti, while it differs in that we apply the Huber function to each component of the modified system (the first and second stage equations in our case) and not to the score of the whole system together. Moreover, we replace the simultaneous estimation of scale and location by simple preliminary scale estimators. These modifications allow us to keep most of the robustness benefit of the Huber function in a context of two-stage estimation, while avoiding calculus complications.

Our contribution in this paper is to develop the 2SH estimator, to derive its asymptotic and small sample properties. This work program is carried out in the case of random regressors and possibly asymmetric errors, weaker restrictions than what is often available in the literature for robust estimation.

The simplicity of the estimation of parameter estimates is one of the 
main motivation of our work, Indeed, the requirement of simplicity is important for practitioners in the context of robust estimation, as stressed for example in Flavin (1999). Our estimator belongs the small class of the available robust estimators for estimation problems with endogenous explanatory variables. It is easily tractable. The practical gain obtained by using a twostage estimation approach and well-known procedures used by practitioners should help contributing to the dissemination of robust methods.

Another interest of our approach is that we keep a simple link with the most popular robust estimator, the Huber estimator, allowing for natural comparisons. Although different methods have been used to obtain robustness in system estimation, the Huber estimator remains a central reference because of its optimal minimax properties (established in Huber, 1964). It has been exploited in more complex frameworks in Krishnakumar and Ronchetti (1997) to derive B-robust estimators. However, Huber and B-robust estimators minimax properties have generally been established only in the case of symmetric errors and small contamination of normal errors. Although these cases are clearly central references, it is less clear if they correspond to the realistic situations of interest for the use of robust estimators. Moreover, B-robust estimators may be complicated to implement, as are variants of the resistant estimators by Krasker and Welsch,. as compared with the use of simple Huber estimators that are available in common statistical package. We deal with this issue by sticking to simple estimation procedures without emphasizing minimax properties that always depend on debatable distribution benchmarks. Other approaches could be to use least-squares or least absolute deviations regressions, which can both be readily implemented in several common statistical packages, as the basis of each estimation stage. Though, with 2SLS substantial robustness issues may arise, while with 2SLAD much efficiency may be lost. Recently, more sophisticated two-stage estimators have been proposed in the framework of quantile regressions and LAD estimators in order to deal with endogeneity of treatment effects (Abadie et al., 2001; Chernozhukov and Hansen, 2001). We do not deal with these sophistications and remain in the usual setting of two-stage estimation methods, in part because we are concerned about robustness and not about treatment effects differing across quantiles. So, the estimator we propose corresponds to a convenient trade-off between simplicity of implementation, efficiency and robustness. However, as most of the other robust estimators in this context, our estimator only protects against local distribution alternatives, but not against large and pervasive departure from normality. The latter situation should be dealt with other methods, for example methods with high breakdown point.

The aim of this paper is to propose and study the $2 \mathrm{SH}$ estimator. In Section 2, we define the model and the estimation method. We derive the asymptotic representation of the $2 \mathrm{SH}$ estimator in Section 3. In Section 4, we analyse the asymptotic normality of the $2 \mathrm{SH}$ estimator. Some Monte Carlo 
simulation experiments are presented in Section 5. Both asymptotic and Monte Carlo results exhibit situations where the 2SH estimator is superior to the 2SLS and to the 2SLAD developed by Amemiya (1982) and Powell (1983). Asymptotic and finite sample results are important because they are first the base of inferences using our estimator, and second because they show that the 2SH estimator is a useful alternative to 2SLS and 2SLAD estimators.

In practice, when there is a suspicion that the data may be contaminated and that the 2SLAD may yield too inefficient estimates (e.g. when the noncontaminated part of the data follows a distribution close to the normal law), then our estimator may provide an interesting procedure for models where some explanatory variables are deemed to be endogenous and when ancillary equations based on exogenous regressors can be used to replace the endogenous regressors of the first stage with their fitted values. Finally, Section 6 concludes. All the technical proofs are collected in the Appendix.

\section{The model and the estimator}

\subsection{The model}

We consider a structural equation given by

$$
y_{t}=Y_{t}^{\prime} \gamma_{0}+x_{1 t}^{\prime} \beta_{0}+u_{t} ; \quad t=1,2, \ldots, T
$$

where $y_{t}$ is the dependent variable, $Y_{t}$ is a $G \times 1$ vector of endogenous variables, $x_{1 t}$ is a $K_{1} \times 1$ vector of random exogenous variables and $u_{t}$ is the error term. We are interested in estimating and making inference on the structural parameters $\alpha_{0}=\left(\gamma_{0}^{\prime}, \beta_{0}^{\prime}\right)^{\prime}$. We denote by $x_{2 t}$ the $K_{2} \times 1$ vector of exogenous variables that are absent from the equation in (1). Let us assume that $Y_{t}^{\prime}=\left(Y_{1 t}, Y_{2 t}, \ldots, Y_{G t}\right)$ admits a reduced-form representation for each $Y_{j t}$ :

$$
Y_{j t}=x_{t}^{\prime} \Pi_{0 j}+V_{j t} \quad ; \quad j=1,2, \ldots, G,
$$

where $x_{t}^{\prime}=\left(x_{1 t}^{\prime}, x_{2 t}^{\prime}\right), \Pi_{0 j}$ is a $K \times 1$ vector of unknown parameters with $K=K_{1}+K_{2}, V_{j t}$ is the error term. Note that (2) can be written as $Y_{t}^{\prime}=x_{t}^{\prime} \Pi_{0}+V_{t}^{\prime}$ where $\Pi_{0}=\left[\Pi_{01}, \Pi_{02}, \ldots, \Pi_{0 G}\right]$ and $V_{t}^{\prime}=\left(V_{1 t}, V_{2 t}, \ldots, V_{G t}\right)$. Then, $y_{t}$ has the following reduced-form representation:

$$
y_{t}=x_{t}^{\prime} \pi_{0}+v_{t}
$$

where

$$
\pi_{0}=\left[\Pi_{0},\left(\begin{array}{c}
I_{K_{1}} \\
0
\end{array}\right)\right] \alpha_{0}
$$


and $v_{t}=u_{t}+V_{t}^{\prime} \gamma_{0}$. Note that (3) can be rewritten as

$$
y_{t}=z_{t}^{\prime} \alpha_{0}+v_{t}
$$

where $z_{t}^{\prime}=x_{t}^{\prime} H\left(\Pi_{0}\right)$ and

$$
H\left(\Pi_{0}\right)=\left[\Pi_{0},\left(\begin{array}{c}
I_{K_{1}} \\
0
\end{array}\right)\right]
$$

is a $K \times\left(G+K_{1}\right)$ matrix. Hence, if the true value of $\Pi_{0}$ were known, the structural parameter vector $\alpha_{0}$ could be directly estimated using (4). The essence of the two-stage approach is to replace $\Pi_{0}$ with an estimator $\hat{\Pi}$ from the first stage. We now turn to the definition of the $2 \mathrm{SH}$ estimator.

\subsection{The estimator}

The reduced-form equation in (2) is used for a first-stage Huber estimation that yields estimator $\hat{\Pi}_{j}$ of $\Pi_{0 j}$, and delivers exogenous predictions of $Y_{j t}$ : $\hat{Y}_{j t}=x_{t}^{\prime} \hat{\Pi}_{j}$. In this situation, the usual Huber estimator would be obtained as the solution to: $\min _{\Pi_{j}} \sum_{t=1}^{T} \rho\left(Y_{j t}-x_{t}^{\prime} \Pi_{j}\right)$, where

$$
\rho(z)=\frac{1}{2} z^{2} 1_{[|z|<k]}+\left(k|z|-\frac{1}{2} k^{2}\right) 1_{[|z| \geq k]},
$$

where $k>0$ is a threshold value fixed in advance. This function, often called the Huber function, has the first derivative

$$
\psi(z)=z 1_{[|z|<k]}+2 k\left(\frac{1}{2}-1_{[z \leq 0]}\right) 1_{[|z| \geq k]} .
$$

However, using the Huber function $\rho(z)$ does not deliver a regression-equivalent estimator. In order to obtain such an estimator, the Huber function must be modified using some scale estimator of the error term. Indeed, the basic Huber estimator, as most M-estimators, is not scale invariant. Following Bickel (1975) and Flavin (1999), we use the standardized median absolute deviation to obtain the required scale estimators as follows:

$$
\begin{aligned}
\hat{\sigma}^{2} & =\operatorname{median}\left\{\left|e_{t}-\operatorname{median}\left\{e_{t}\right\}\right|\right\} / \Phi^{-1}(3 / 4) \\
\hat{\sigma}_{j}^{2} & =\text { median }\left\{\left|e_{j t}-\operatorname{median}\left\{e_{j t}\right\}\right|\right\} / \Phi^{-1}(3 / 4) \\
j & =1,2, \ldots, G
\end{aligned}
$$

where $e_{t}$ are the residuals obtained from the LS regression of $y_{t}$ on $x_{t}$ and $e_{j t}$ are the residuals from the LS regression of $Y_{j t}$ on $x_{t}$. Using a preliminary scale estimate is possible in our problem because it is based on consistent fitted values. Then, $\hat{\sigma}^{2}$ and $\hat{\sigma}_{j}^{2}$ are consistent. In these conditions, plugging 
them into the optimisation program defining Huber estimator does not affect the consistency of the estimation procedure.

For symmetric distributions, the median absolute deviation (MAD) is asymptotically equivalent to half the interquartile range, is minimax with respect to bias and has the best breakdown properties under $\varepsilon$-contamination in the class of M-estimators (Huber, 1981). Note that this choice of scale estimator is different from Huber proposal ${ }^{2}$, or from B-estimators that follow Huber approach (Krishnakumar and Ronchetti, 1997). The advantage of Huber and B-estimator approaches is to ensure minimax properties for both scale and location parameters, that is, to minimise the variance of these estimators under a 'least-informative distribution' of the contaminated errors. The drawback of this approach is to lead to unattractive calculus complication, perhaps one reason why these estimators are relatively little used despite their interesting properties. One of the calculus complication comes from the simultaneous estimation of the location and the scale. Moreover, the optimality of these location and scale estimators is only reached when the reference distribution is the 'least-informative one', and not for other alternatives that may be more plausible. Meanwhile, the minimax properties refer to symmetric contamination and normal uncontaminated errors. Finally, minimax approaches may not protect as well against heavy-tails errors that may be more likely than 'least-informative distributions'. Since the breakdown properties of simultaneous estimators of scale and location, or of estimators with preliminary scaling, are mainly determined by the breakdown properties of the scale estimator (See Huber 1981), it seems a good idea to use the MAD as scale estimator. Indeed, the MAD has the largest breakdown point $(1 / 2)$ of all M-estimators. So, we explore another route in this paper, namely using the MAD as preliminary scale estimator of each stage of a two-stage estimation context to deal with endogeneity issues. Another reason to consider the MAD is that it can be considered as the "most robust estimator of scale' (see Huber, 1981, p122) in the sense that it is the limit of the Huber estimator of scale when the contamination grows up to almost all the observations.

Then, the first-stage Huber estimator $\hat{\Pi}_{j}$ is defined as the solution to the following:

$$
\min _{\Pi_{j}} \sum_{t=1}^{T} \rho_{\hat{\sigma}_{j}}\left(Y_{j t}-x_{t}^{\prime} \Pi_{j}\right),
$$

where $\rho_{\hat{\sigma}_{j}}(z)=\rho\left(z / \hat{\sigma}_{j}\right)$. The corresponding first-order condition is:

$T^{-1 / 2} \sum_{t=1}^{T} x_{t} \psi_{\hat{\sigma}_{j}}\left(Y_{j t}-x_{t}^{\prime} \hat{\Pi}_{j}\right)=0$ for $j=1, \ldots, G$, where $\psi_{\hat{\sigma}_{j}}(z)=\psi\left(z / \hat{\sigma}_{j}\right)$, dropping the factor $1 / \hat{\sigma}_{j}$.

\footnotetext{
${ }^{2}$ An M-estimator $S$ of scale defined by $\int \chi(x / S(F)) F(d x)=0$ with $\chi(x)=x^{2}-\beta$ for $|x| \leq \beta$ and $\chi(x)=k^{2}-\beta$ for $|x|>\beta$, with $\beta$ such that $\int \chi(x) \Phi(d x)=0$, all with Huber's obvious original notation.
} 
Let us now turn to the second stage of the estimation. The 2SH estimator $\hat{\alpha}$ of $\alpha_{0}$ is the solution to the following minimisation programme:

$$
\min _{\alpha} S_{T}(\alpha, \hat{\Pi})=\sum_{t=1}^{T} \rho_{\hat{\sigma}}\left(y_{t}-\hat{x}_{t}^{\prime} \alpha\right),
$$

where, $\hat{x}_{t}^{\prime}=x_{t}^{\prime} H(\hat{\Pi})$ with $\hat{\Pi}=\left[\hat{\Pi}_{1}, \hat{\Pi}_{2}, \ldots, \hat{\Pi}_{G}\right]$ incorporates the first-stage predictions of the endogenous variables. Alternatively, the $2 \mathrm{SH}$ estimator can be defined as a solution to the first-order condition: $\sum_{t=1}^{T} H(\hat{\Pi})^{\prime} x_{t} \psi_{\hat{\sigma}}\left(y_{t}-\right.$ $\left.\hat{x}_{t}^{\prime} \alpha\right)=0$. The same Huber estimator (i.e. the same parameter $k$ ) is used for both stages consistently with our requirement of simplicity. Alternatively, different values for $k$ could be used for the two stages, little changing the properties of the estimator. In the next section, we derive the asymptotic representation of the $2 \mathrm{SH}$ estimator. The following conditions are needed for this task.

Assumption 1. (i) The entire sequence $\left\{\left(v_{t}, V_{t}, x_{t}\right)\right\}$ is independent and identically distributed with $\sigma^{2}=E\left(v_{t}^{2}\right) \in(0, \infty), \sigma_{j}^{2}=E\left(V_{j t}^{2}\right) \in(0, \infty)$ and $\sigma_{u}^{2}=E\left(v_{t}^{2}\right) \in(0, \infty)$.

(ii) $E\left(\left\|x_{t}\right\|\right)^{3}<\infty$ where $\left\|x_{t}\right\|=\left(x_{t}^{\prime} x_{t}\right)^{1 / 2}$.

Assumption 2. (i) $v_{t}$ has a conditional cdf $F(\cdot \mid x)$ that is Lipschitz continuous for all $x$.

(ii) $E\left[\psi_{\sigma}\left(v_{t}\right) \mid x_{t}\right]=0$.

(iii) $Q=E\left\{g\left(0 \mid x_{t}\right) x_{t} x_{t}^{\prime}\right\}$ is finite and positive-definite where $g\left(0 \mid x_{t}\right)=$ $G^{\prime}\left(0 \mid x_{t}\right)$ with $G\left(z \mid x_{t}\right)=E\left[\psi_{\sigma}\left(v_{t}+z\right) \mid x_{t}\right]$.

(iv) $H\left(\Pi_{0}\right)$ is of full column rank.

The iid condition in Assumption 1(i), which may be interpreted as a description of the sampling scheme, is imposed for presentation simplicity. It can be relaxed to include heteroskedasticity and serial correlation. In such a case, our covariance matrix in Proposition 2 further on should be modified by using the Newey-West type covariance matrix. Assumption 1(ii) is the moment condition on the vector of exogenous variables. It is useful to establish the asymptotic representation of all considered estimators by applying a theorem of stochastic equicontinuity for the relevant empirical process. We also use it to obtain the boundedness of the asymptotic covariance matrix of parameter estimates. This condition and the other conditions of the exogenous regressors are weaker that what is sometimes done in two-stage estimation papers, where the exogenous regressors are assumed fixed (e.g. in Powell, 1983).

Assumption 2(i) simplifies the demonstration of convergence of certain remainder terms to zero. It is used to derive the asymptotic representation 
of our estimator. Assumption 2(ii) says that the trimmed conditional mean of $v_{t}$ is zero. If $\psi$ were instead the quantile function (as in Powell, 1978), it would mean that the conditional quantile of $v$ is zero. In the case of OLS, $\psi$ would be the identity and the restriction would correspond to the nullity of the conditional mean. Assumption 2(ii) is satisfied if $v_{t}$ is symmetric and $E\left(v_{t} \mid x_{t}\right)=0$, which is commonly assumed for Huber estimators (e.g. Bickell, 1975, Carroll and Ruppert, 1982). Here, even if the distribution of $v_{t}$ is not symmetric, when the conditional density of $v_{t}$ is not equal to zero over a large support and when there is an intercept in the model, this condition can be considered as an inoccuous normalisation of the intercept. Therefore, it should be satisfied in applications where there are intercepts in the model equations, the usual case. Indeed, in that case $E\left(\psi\left(v_{t}\right) \mid x_{t}\right)=0$ and $E\left(\psi\left(v_{t}\right) \mid x_{t}\right) \neq 0$ correspond to isomorphic statistical structures that distinguish themselves only by the value of the intercept term. They are observationally equivalent structures. Therefore, it is possible to impose $E\left(\psi(v) \mid x_{t}\right)=0$, and thus to fix the value of the intercept, without loss of generality. Assumption (iv) is an identification condition, which is standard for simultaneous equations models.

Assumption A2(iii) is the counterpart of the usual condition for OLS that the mean cross-product of the regressor vectors converges towards a finite positive definite matrix. Here, the cross-product matrix is weighted by a coefficient that characterises how fast the trimmed conditional mean of $v_{t}$ changes around zero along the change in $v_{t}$. This conditions is necessary for consistency and for the inversion of the relevant empirical process in order to establish the asymptotic normality. We do not need conditions about the densities of $v_{t}$ and $V_{j t}$ around zero because such conditions are implied by the condition of positive definiteness of matrices $Q$ and $Q_{j}$.

The structural equation in (1) is identified if the number of zero restrictions $\left(K_{2}\right)$ is not less than the number of endogenous variables $(G)$. Noting that $H\left(\Pi_{0}\right)$ is a $\left(K_{1}+K_{2}\right) \times\left(K_{1}+G\right)$ matrix, Assumption (iv) implies that $K_{2} \geq G$, which includes the exact identified case $\left(K_{2}=G\right)$ and the overidentified case $\left(K_{2}>G\right)$. This assumption is needed when proving that the 2SH estimator $\hat{\alpha}$ is consistent for the true parameter $\alpha_{0}$.

It is now time to return to the consequences of the possible endogeneity of $Y_{t}$. If the $Y_{t}^{\prime} / s$ are exogenous in the general sense, we have $E\left[\psi_{\sigma_{u}}\left(u_{t}\right) \mid Y_{t}, x_{t}\right]=$ 0 where $\psi_{\sigma_{u}}(z)=\psi\left(z / \sigma_{u}\right)$ and $\sigma_{u}$ is the preliminary scale estimator. Then, the truncation of outliers, via the Huber function, in terms of the error terms makes intuitive sense. However, if the $Y_{t} / s$ are endogenous, we expect in general that $E\left[\psi_{\sigma_{u}}\left(u_{t}\right) \mid Y_{t}, x_{t}\right] \neq 0$ as a consequence of the non-separation of the marginal laws of the $Y_{t} / s$ and of the $u_{t} / s$. It is unclear what the truncation of the error terms means in this situation, and therefore what is estimated. Our 2SH estimator ensures that at each stage of the estimation, the truncation eliminating outliers applies to well-defined error terms that can be interpreted as prediction errors from a set of exogenous variables. 


\section{The asymptotic representation}

As in Hjort and Pollard (1993), consistency and asymptotic normality can be delivered in one unique step. For this, we derive the asymptotic derivation of the estimator based on the following empirical process $M_{T}(\cdot)$ :

$$
M_{T}(\Delta)=T^{-1 / 2} \sum_{t=1}^{T} x_{t} \psi_{\sigma}\left(v_{t}-T^{-1 / 2} x_{t}^{\prime} \Delta\right)=T^{-1 / 2} \sum_{t=1}^{T} m_{\sigma}\left(w_{t}, \Delta\right),
$$

where $\Delta$ is a $K \times 1$ vector, $w_{t}=\left(v_{t}, x_{t}^{\prime}\right)^{\prime}$ and $m_{\sigma}\left(w_{t}, \Delta\right)=x_{t} \psi_{\sigma}\left(v_{t}-\right.$ $\left.T^{-1 / 2} x_{t}^{\prime} \Delta\right)$. We can apply Theorems 1-3 in Andrews (1994) because of the bounded variations of function $\psi_{\sigma}$, of the iid condition in Assumption 1(i) and of the moment condition on $x_{t}$ in Assumption 1(ii). As a result, we have the following preliminary lemma.

Lemma 1 Suppose that Assumptions 1 and 2(i) hold. Then, for some finite $L>0$, we have the following:

$$
\sup _{\|\Delta\| \leq L}\left\|M_{T}(\Delta)-M_{T}(0)+Q \Delta\right\|=o_{p}(1) .
$$

Lemma 1 together with the remaining conditions in Assumption 2 is used to obtain the following asymptotic representation for the $2 \mathrm{SH}$ estimator.

Proposition 1 Suppose that Assumptions 1 and 2 hold and that $T^{1 / 2}(\hat{\Pi}-$ $\left.\Pi_{0}\right)=O_{p}(1)$. Then, we have the following:

$$
\begin{aligned}
& T^{1 / 2}\left(\hat{\alpha}-\alpha_{0}\right) \\
= & Q_{z z}^{-1} H\left(\Pi_{0}\right)^{\prime}\left\{T^{-1 / 2} \sum_{t=1}^{T} x_{t} \psi_{\sigma}\left(v_{t}\right)-Q T^{1 / 2}\left(\hat{\Pi}-\Pi_{0}\right) \gamma_{0}\right\}+o_{p}(1),
\end{aligned}
$$

where $Q_{z z}=H\left(\Pi_{0}\right)^{\prime} Q H\left(\Pi_{0}\right)$.

In the above proposition, a high level condition on $\hat{\Pi}$ has been imposed, namely $T^{1 / 2}\left(\hat{\Pi}-\Pi_{0}\right)=O_{p}(1)$. This condition can be seen as a consequence of the following Assumption 3.

Assumption 3. (i) $V_{j t}$ has a conditional cdf $H_{j}(\cdot \mid x)$ for $j=1, \ldots, G$ that is Lipschitz continuous for all $x$.

(ii) $E\left[\psi_{\sigma_{j}}\left(V_{j t}\right) \mid x_{t}\right]=0$ for $j=1, \ldots, G$, where $\psi_{\sigma_{j}}(z)=\psi\left(z / \sigma_{j}\right)$

(iii) $Q_{j}=E\left\{g_{j}\left(0 \mid x_{t}\right) x_{t} x_{t}^{\prime}\right\}$ are finite and positive-definite, $j=1, \ldots, G$, where $g_{j}\left(0 \mid x_{t}\right)=G_{j}^{\prime}(0)$ with $G_{j}(z)=E\left[\psi_{\sigma_{j}}\left(V_{j t}+z\right) \mid x_{t}\right]$.

Assumptions 3(i)-(iii) are similar to Assumptions 2(i)-(iii). So, the same justification applies. We start the analysis of the asymptotic properties of 
the $2 \mathrm{SH}$ estimator with the derivation of its asymptotic representation from which we shall deduce the asymptotic normality and the asymptotic covariance matrix. It can easily be shown that $g\left(0 \mid x_{t}\right)=F\left(k \mid x_{t}\right)-F\left(-k \mid x_{t}\right)$ and $g_{j}\left(0 \mid x_{t}\right)=H_{j}\left(k \mid x_{t}\right)-H_{j}\left(-k \mid x_{t}\right)$ for $j=1, \ldots, G$. To simplify we eliminate from now the conditioning from the notations for functions $G, G_{j}, g, g_{j}$.

Proposition 2 Suppose that Assumptions 1-3 hold. Then, the 2SH estimator $\hat{\alpha}$ has the asymptotic representation:

$$
T^{1 / 2}\left(\hat{\alpha}-\alpha_{0}\right)=D T^{-1 / 2} \sum_{t=1}^{T} Z_{t}+o_{p}(1)
$$

where $D=Q_{z z}^{-1} H\left(\Pi_{0}\right)^{\prime}\left[I,-Q Q_{1}^{-1} \gamma_{01}, \ldots,-Q Q_{G}^{-1} \gamma_{0 G}\right], Z_{t}=W_{t} \otimes x_{t}$, and $W_{t}=\left[\psi_{\sigma}\left(v_{t}\right), \psi_{\sigma_{1}}\left(V_{1 t}\right), \ldots, \psi_{\sigma_{G}}\left(V_{G t}\right)\right]^{\prime}$.

Let us now compare this result with the following asymptotic representations of $2 \mathrm{SLS}$, denoted $\hat{\alpha}_{2 S L S}$, and $2 \mathrm{SLAD}$, denoted $\hat{\alpha}_{2 S L A D}$. It is easy to show under some regularity conditions ${ }^{3}$ comparable to Assumptions 1-3 that

$$
T^{1 / 2}\left(\hat{\alpha}_{2 S L S}-\alpha_{0}\right)=D^{L S} T^{-1 / 2} \sum_{t=1}^{T} Z_{t}^{L S}+o_{p}(1),
$$

where $D^{L S}=Q_{z z}^{L S-1} H\left(\Pi_{0}\right)^{\prime}\left[I,-\gamma_{01} I, \ldots,-\gamma_{0 G} I\right], Q_{z z}^{L S}=H\left(\Pi_{0}\right)^{\prime} Q^{L S} H\left(\Pi_{0}\right)$, $Q^{L S}=E\left\{x_{t} x_{t}^{\prime}\right\}$ and $Z_{t}^{L S}=W_{t}^{L S} \otimes x_{t}$, and $W_{t}^{L S}=\left[v_{t}, V_{1 t}, \ldots, V_{G t}\right]^{\prime}$. Moreover,

$$
T^{1 / 2}\left(\hat{\alpha}_{2 L A D}-\alpha_{0}\right)=D^{L A D} T^{-1 / 2} \sum_{t=1}^{T} Z_{t}^{L A D}+o_{p}(1),
$$

where

$$
\begin{aligned}
& D^{L A D}=Q_{z z}^{L A D-1} H\left(\Pi_{0}\right)^{\prime}\left[I,-Q^{L A D}\left(Q_{1}^{L A D}\right)^{-1} \gamma_{01}, \ldots,-Q^{L A D}\left(Q_{G}^{L A D}\right)^{-1} \gamma_{0 G}\right], \\
& Q_{z z}^{L A D}=H\left(\Pi_{0}\right)^{\prime} Q^{L A D} H\left(\Pi_{0}\right), Q^{L A D}=E\left\{f\left(0 \mid x_{t}\right) x_{t} x_{t}^{\prime}\right\}, \\
& Q_{j}^{L A D}=E\left\{h_{j}\left(0 \mid x_{t}\right) x_{t} x_{t}^{\prime}\right\}, \text { for } j=1, \ldots, G, Z_{t}^{L A D}=W_{t}^{L A D} \otimes x_{t}, \\
& W_{t}^{L A D}=\left[\psi_{L A D}\left(v_{t}\right), \psi_{L A D}\left(V_{1 t}\right), \ldots, \psi_{L A D}\left(V_{G t}\right)\right], \\
& \psi_{L A D}(z)=0.5-1[z \leq 0], \text { and } f(\cdot \mid x) \text { and } h_{j}(\cdot \mid x) \text { are the conditional }
\end{aligned}
$$
pdf's of $v_{t}$ and $V_{j t}$ respectively.

\footnotetext{
${ }^{3}$ The complete list of regularity conditions can be found in Kim and Muller (2004). Among the regularity conditions, the crucial ones that ensure the consistency of the 2SLS and 2SLAD estimators are $E\left[v_{t} \mid x_{t}\right]=0, E\left[V_{j t} \mid x_{t}\right]=0, E\left[\psi_{L A D}\left(v_{t}\right) \mid x_{t}\right]=0$, and $E\left[\psi_{L A D}\left(V_{j t}\right) \mid x_{t}\right]=0$. They are essentially two differences in the assumptions for the consistency and asymptotic normality of 2SLS, 2SLAD and 2SH: first, the semiparametric restriction, second the existence and positive definiteness of matrices incorporating crossproducts of regressions. The only differences for these matrices lies in the presence of a different weighing factors. It does not seem to us that this fundamentally changes the scope of cases to consider. As for the semiparametric restriction, as long as the support of the errors is broad enough, and that there is an intercept term in the model, they can all be considered as mere normalisation of the intercept coefficient, and the slope estimates can be validly compared.
} 


\section{Asymptotic normality and covariance matrix}

We now show the asymptotic normality of the $2 \mathrm{SH}$ estimator by applying the Lindeberg-Levy CLT.

Proposition 3 Suppose that Assumptions 1-3 hold. Then,

$$
T^{1 / 2}\left(\hat{\alpha}-\alpha_{0}\right) \stackrel{d}{\rightarrow} N\left(0, D \Omega D^{\prime}\right),
$$

where $\Omega=E\left(W_{t} W_{t}^{\prime} \otimes x_{t} x_{t}^{\prime}\right)$.

These asymptotic results show the differences in the asymptotic properties of 2SH, 2SLS and 2SLAD. The proof of the asymptotic representation for the 2SLAD with random regressors can be found for example in Kim and Muller (2004). The differences in assumptions in part boil down to different normalisation rules (of the type $E\left[\psi\left(v_{t}\right) \mid x_{t}\right]=0$ ) for the intercept, which we assume from now. So, the comparison makes sense for the slope coefficients at least, which are generally the coefficients of interest for applied researchers since they convey the effects of the explanatory variables. First, the influence function of 2SLS, easy to read from the empirical processes of the asymptotic representation, is not bounded, as opposed to that of $2 \mathrm{SH}$ and of 2SLAD that are robust methods. Then, the 2SLS is not appropriate for example when the errors are seriously contaminated. Second, for each estimation method it is possible to exhibit corresponding error distributions for which the method is efficient and dominates the other two estimation methods: Normal distributions for 2SLS, Laplace distributions for 2SQR and minimax Huber distributions for 2SH estimator (Huber, 1964). Therefore, no method can be considered to be best in all cases in terms of efficiency and $2 \mathrm{SH}$ inherits minimax properties from the Huber estimator. 2SLAD is known to suffer from particularly low efficiency in situations close to the Gaussian case. Also, it is apparent that low values of $f\left(0 \mid x_{t}\right)$ and $h_{j}\left(0 \mid x_{t}\right)$ for 2SLAD can degrade the efficiency of these estimators. On the whole, 2SH appears as an interesting compromise between the properties of robustness of the 2SLAD and the efficiency of 2SLS under normality. In the next section, we present a simulation study that enables us to compare small sample properties of $2 \mathrm{SH}$ with that of 2SLS and 2SLAD.

\section{Monte Carlo simulation}

Using Monte-Carlo simulations, we examine the small sample behaviour of the Huber estimator for the structural parameters $\left(\gamma_{0}, \beta_{0}\right)$, on the one hand when the endogeneity problem is ignored and on the other hand when the problem is treated by using the $2 \mathrm{SH}$ estimator. We also compare this behaviour with that of 2SLS and 2SLAD in small samples. 
We base our simulations on the simplest possible model: a simultaneous equation system with two simple equations so as to be able to naturally inject endogeneity into the model. The first equation, which is the equation of interest, contains two endogenous variables (including the dependent variable) and two exogenous variables including a constant. Four exogenous variables are present in the whole system.

The structural simultaneous equation system can then be written

$$
B\left[\begin{array}{c}
y_{t} \\
Y_{t}
\end{array}\right]+\Gamma x_{t}=U_{t}
$$

where $\left[\begin{array}{c}y_{t} \\ Y_{t}\end{array}\right]$ is a $2 \times 1$ vector of endogenous variables, $x_{t}$ is a $4 \times 1$ vector of exogenous variables with the first element equal to one. Then, we have an intercept term in the model and the semiparametric restrictions of the three estimators correspond to three different normalisation of the intercept coefficient. Therefore, we are mostly interested in the comparison of the slope coefficients. $U_{t}$ is a $2 \times 1$ vector of error terms. We specify the structural parameters as follows: $B=\left[\begin{array}{cc}1 & -0.5 \\ -0.7 & 1\end{array}\right]$ and $\Gamma=\left[\begin{array}{llll}-1 & -0.2 & 0 & 0 \\ -1 & 0 & -0.4 & -0.5\end{array}\right]$. The system is over-identified by the zero restrictions $\Gamma_{13}=\Gamma_{14}=\Gamma_{22}=0$. Hence, the first equation is

$$
y_{t}=Y_{t} \gamma+x_{1 t}^{\prime} \beta+u_{t}=0.5 Y_{t}+1+0.2 x_{12 t}+u_{t}
$$

where $\gamma=-B_{12}=0.5, \beta^{\prime}=\left(\beta_{0}, \beta_{1}\right)=\left(-\Gamma_{11},-\Gamma_{12}\right)=(1,0.2), x_{1 t}$ is the $2 \times 1$ subvector consisting of the first two elements of $x_{t}\left(1\right.$ and $\left.x_{12 t}\right)$, and $u_{t}$ is the first element of $U_{t}$. The second equation is therefore

$$
Y_{t}=0.7 y_{t}+1+0.4 x_{13 t}+0.5 x_{14 t}+w_{t}
$$

where $x_{13 t}$ and $x_{14 t}$ are respectively the third and fourth elements of $x_{t}$, and $w_{t}$ is the second element of $U_{t}$.

The choice of the parameter values is led by the following considerations. Since we are not so much interested in the intercepts, we simply choose them to be equal to 1 for all equations and all normalisations. Only slightly attenuated effects are chosen for the cross effects of the two endogenous variables (coefficients 0.5 and 0.7 ) so that the endogeneity be interesting but not extreme. Identification restrictions and overidentification drive the appearance of exogenous variables in the equations. Moderate but nonnegligible and relatively comparable effects are allowed for these variables (coefficients $0.2,0.4,0.5$ ). In that way, a balanced situation is obtained where endogenous and exogenous influences should all matter. Thus, we expect that all parameter values in $B$ and $\Gamma$ play important roles, except 
perhaps for the intercept parameter, and especially the interaction between endogenous variables.

We can rewrite the system in a matrix representation $\left[\begin{array}{cc}y & Y\end{array}\right] B^{\prime}=$ $-X \Gamma^{\prime}+U$, which yields the reduced-form equations $\left[\begin{array}{ll}y & Y\end{array}\right]=X\left[\begin{array}{ll}\pi_{0} & \Pi_{0}\end{array}\right]+$ $\left[\begin{array}{ll}v & V\end{array}\right]$, where $\left[\begin{array}{ll}\pi_{0} & \Pi_{0}\end{array}\right]=-\Gamma^{\prime}\left(B^{\prime}\right)^{-1}$ and $\left[\begin{array}{ll}v & V\end{array}\right]=U\left(B^{\prime}\right)^{-1}$. Using $\left[\begin{array}{ll}\pi_{0} & \Pi_{0}\end{array}\right]=-\Gamma^{\prime}\left(B^{\prime}\right)^{-1}$, we obtain $\pi_{0}^{\prime}=(2.3,0.3,0.3,-0.15)$ and $\Pi_{0}^{\prime}=$ $(2.6,0.2,0.6,-0.3)$.

The errors $\left[\begin{array}{ll}v & V\end{array}\right]$ are drawn from the standard normal $N(0,1)$, the Student-t with 3 degrees of freedom, t(3), and the Lognormal errors with $\log$-mean $\mu=0$ and log-standard deviation $\sigma=1$, denoted by $L N(0,1)$, in such a way that Assumptions 2(ii) and 3(ii) are satisfied. We draw the second to fourth columns in $X$ from the normal distribution with mean $(0.5,1,-0.1)^{\prime}$, variances equal to $1, \operatorname{cov}\left(x_{2}, x_{3}\right)=0.3, \operatorname{cov}\left(x_{2}, x_{4}\right)=0.1$ and $\operatorname{cov}\left(x_{3}, x_{4}\right)=0.2$. The variances are chosen equal to 1 for normalisation purposes, while the correlations between the exogenous variables are neither extreme nor negligible. Given $X,\left[\begin{array}{ll}v & V\end{array}\right]$ and $\left[\begin{array}{ll}\pi_{0} & \Pi_{0}\end{array}\right]$, we generate the endogenous variables $\left[\begin{array}{ll}y & Y\end{array}\right]$ by using the reduced-form equations.

We use 1000 replications and the sample size is of 50. For each replication, we estimate the parameter values $\gamma$ and $\beta=\left(\beta_{0}, \beta_{1}\right)^{\prime}$ and we calculate the deviation of the estimates from the true values. Then, we compute the sample mean and sample standard deviation of those deviations based on the 1000 replications.

The simulated means and simulated standard deviation for the estimated $\gamma, \beta_{0}$ and $\beta_{1}$ of the one-stage Huber estimator, the 2SH, the 2SLS and the 2SLAD are displayed in Table 1 for Gaussian errors, Table 2 for $t(3)$ errors and Table 3 for $\operatorname{LN}(0,1)$ errors. The tables show the deviations from the true value for the simulated means. For Huber estimator, we use two different values of parameter $k(k=2$ or $k=4)$.

As mentioned above, we are mostly interested in the slope coefficients since strictly speaking the intercept coefficients are not comparable across methods. However, it remains interesting to confront the accuracy of the various intercept estimators even when corresponding to different normalisations. Indeed, the order of magnitude of these estimates should be similar since they all represent central tendencies.

For all three parameters, the Huber estimator is characterised by a systematic bias in finite samples, which does not vanish as the sample size increases from 50. By contrast, the means of the 2SH, 2SLS and 2SLAD estimates (in deviation to the true value) are much closer to zero than the means of the one-stage Huber estimator for the two considered distributions. The endogeneity problem appears well corrected by the $2 \mathrm{SH}$ even with small samples in the studied cases.

As expected, for the Gaussian errors, the 2SLS performs better than the 2SLAD. Indeed, the 2SLS is very close to the $2 \mathrm{SH}$ for $k=2$ (2SLS is only 
marginally more precise) and is almost identical to $2 \mathrm{SH}$ for $k=4$. In the case of the Student errors, the 2SLS is clearly less accurate than the $2 \mathrm{SH}$ and than the 2SLAD that are less sensitive to heavy tails. Meanwhile, the simulated means for the $2 \mathrm{SH}$ are less precise than that obtained with the 2SLAD for $k=4$, but the reverse result is observed for $k=2$. In the case of the Lognormal distribution, two interesting observations emerge. First, as expected for all two-step estimators, only the intercept estimate $\left(\hat{\beta}_{0}\right)$ becomes biased. However, as we mentioned above, the bias of the different estimators are not comparable since they refer to different central tendencies. Secondly, when considering the remaining unbiased components $\left(\hat{\gamma}\right.$ and $\left.\hat{\beta}_{1}\right)$, the 2SH estimator is substantially more accurate than the 2SLS and the 2SLAD for $k=2$, while only marginally better for $k=4$. Then, adjusting parameter $k$ may help to tune in the efficiency-robustness properties of $2 \mathrm{SH}$ as compared with 2SLS and 2SLAD. This is an additional advantage of $2 \mathrm{SH}$, which could be systematically investigated to yield a rule for fixing $k$ in different cases of interest.

Clearly, there exist cases easy to exhibit where 2SH dominates 2SLS or 2SLAD in terms of variance of estimated slope parameters, even without introducing arbitrary outliers. This supports the contention that $2 \mathrm{SH}$ is a useful complement to the toolbox of two-stage estimation methods for systems of equations with endogeneity problems.

\section{Conclusion}

In this article we propose and study the Two-Stage Huber (2SH) estimator in the case of random regressors and possibly asymmetric errors. The error scale is corrected by preliminary uses of the Median Absolute Deviation estimator at every stages. We derive the formula of the asymptotic covariance matrix for the parameters of interest. The comparison of the asymptotic properties and of Monte Carlo simulation results for the 2SH estimator, the 2SLS estimator and the 2SLAD estimator indicates that none of these estimators dominates the other ones even for a few simple distributions, whether asymptotically or for finite samples. In this situation, the $2 \mathrm{SH}$ estimator provides a convenient compromise between requirements of simplicity of implementation, robustness and efficiency. Asymptotic and finite sample results are important because they are first the base of inferences using our estimator, and second because they show that the $2 \mathrm{SH}$ estimator is a useful alternative to 2SLS and 2SLAD estimators.

In practice, when there is a suspicion that the data may be contaminated and that the 2SLAD may yield too inefficient estimates (e.g. if the noncontaminated part of the data follows a distribution close to the normal law), then our estimator may provide an interesting procedure for models where some explanatory variables are deemed to be endogenous and when 
ancillary equations based on exogenous regressors can be used to replace the endogenous regressors of the first stage with their fitted values.

A practical difficulty however, as for any other two-stage method, is that specific calculations must be carried out to estimate the asymptotic covariance matrix of the parameters. Arguably, the formula of the matrix is complex enough. However, convenient estimators of this matrix can be obtained by plugging residual and nonparametrix estimators of expressions involving densities, similarly to what is done in Kim and Muller (2004) for two-stage quantile regressions. Alternatively, bootstrap estimators of the covariance matrix could be explored, perhaps on the lines proposed by Hahn (1995) for quantile regressions. Finally, an other useful extension would be the analysis of the inaccuracy coming from the preliminary scale estimation for each stage.

\section{Appendix}

Proof of Lemma 1. We first define $V_{T}(\Delta)=T^{-1 / 2} \sum_{t=1}^{T}\left[m_{\sigma}\left(w_{t}, \Delta\right)-\right.$ $\left.E\left\{m_{\sigma}\left(w_{t}, \Delta\right)\right\}\right]=M_{T}(\Delta)-E\left(M_{T}(\Delta)\right)$. In order to apply Theorem 1 in Andrews (1994) to the empirical process $V_{T}(\Delta)$, we need to check the following two conditions: (i) $m\left(w_{t}, \Delta\right)$ satisfies Pollard's entropy condition with some envelop $\bar{M}\left(w_{t}\right)$; (ii) For some $\delta>2, \lim _{T \rightarrow \infty} T^{-1} \sum_{t=1}^{T} E\left[\left\{\bar{M}\left(w_{t}\right)\right\}^{\delta}\right]<\infty$.

We define $f_{1}\left(w_{t}, \Delta\right)=x_{t}$ and $f_{2}\left(w_{t}, \Delta\right)=\psi_{\sigma}\left(v_{t}-T^{-1 / 2} x^{\prime} \Delta\right)$ so that $m_{\sigma}\left(w_{t}, \Delta\right)=f_{1}\left(w_{t}, \Delta\right) f_{2}\left(w_{t}, \Delta\right)$. We note that each element of $f_{1}\left(w_{t}, \Delta\right)$ is Type I class with envelope $\left\|x_{t}\right\|$ (see Andrews, 1994, for the definition of Type I class) and $f_{2}\left(w_{t}, \Delta\right)$ is also Type I class with envelope $C=1 \vee k$ where $\checkmark$ is the maximum operator. Hence, the product $m_{\sigma}\left(w_{t}, \Delta\right)$ satisfies Pollard's entropy condition with envelope $\bar{M}\left(w_{t}\right)=C\left(\left\|x_{t}\right\| \vee 1\right)$ by Theorem 3 in Andrews (1994), which ensures the first condition. By Assumption 1(i) we have $\lim _{T \rightarrow \infty} T^{-1} \sum_{t=1}^{T} E\left[\left\{\bar{M}\left(w_{t}\right)\right\}^{\delta}\right]=C^{\delta} E\left(\left\|x_{t}\right\| \vee 1\right)^{\delta}$, which is bounded by Assumption 1(ii). Hence, by applying Theorem 1 in Andrews (1994), we obtain the following:

$$
\sup _{\left\|\Delta_{1}-\Delta_{2}\right\| \leq L}\left\|V_{T}\left(\Delta_{1}\right)-V_{T}\left(\Delta_{2}\right)\right\|=o_{p}(1)
$$

for some finite $L>0$. Setting $\Delta_{1}=\Delta$ and $\Delta_{2}=0$ in (6) yields

$$
\sup _{\|\Delta\| \leq L}\left\|M_{T}(\Delta)-M_{T}(0)-\left\{E\left(M_{T}(\Delta)\right)-E\left(M_{T}(0)\right)\right\}\right\|=o_{p}(1) .
$$

We now show that $E\left(M_{T}(\Delta)\right)-E\left(M_{T}(0)\right) \rightarrow-Q \Delta$. Using the law of 
iterated expectation and the mean value theorem, we have that

$$
\begin{aligned}
& E\left(M_{T}(\Delta)\right)-E\left(M_{T}(0)\right) \\
= & E\left[T^{-1 / 2} \sum_{t=1}^{T} x_{t}\left\{G\left(-T^{-1 / 2} x_{t}^{\prime} \Delta \mid x_{t}\right)-G\left(0 \mid x_{t}\right)\right\}\right] \\
= & -E\left\{T^{-1} \sum_{t=1}^{T} \frac{G\left(-T^{-1 / 2} x_{t}^{\prime} \Delta \mid x_{t}\right)-G\left(0 \mid x_{t}\right)}{-T^{-1 / 2} x_{t}^{\prime} \Delta} x_{t} x_{t}^{\prime}\right\} \Delta \\
= & -E\left\{T^{-1} \sum_{t=1}^{T} g\left(\xi_{T} \mid x_{t}\right) x_{t} x_{t}^{\prime}\right\} \Delta,
\end{aligned}
$$

where $\xi_{T, t}$ is between zero and $-T^{-1 / 2} x_{t}^{\prime} \Delta$. Since one can show that $g(\lambda \mid x)=$ $\sigma^{-1}\{F(-\lambda+\sigma k \mid x)-F(-\lambda-\sigma k \mid x)\}$, Assumption 2(i) implies that $g(\lambda \mid x)$ is Lipschitz continuous in $\lambda$ for all $x$; that is, for some constant $L_{0} \in(0, \infty)$

and for all $x,\left|g\left(\lambda_{1} \mid x\right)-g\left(\lambda_{2} \mid x\right)\right| \leq L_{0}\left|\lambda_{1}-\lambda_{2}\right|$. Now we consider the $(i, j)^{t h}$ element of $\left|E\left\{T^{-1} \sum_{t=1}^{T} g\left(\xi_{T} \mid x_{t}\right) x_{t} x_{t}^{\prime}\right\}-E\left\{g\left(0 \mid x_{t}\right) x_{t} x_{t}^{\prime}\right\}\right|$, which is given by

$$
\begin{aligned}
& \left|E\left\{T^{-1} \sum_{t=1}^{T}\left(g\left(\xi_{T} \mid x_{t}\right)-g\left(0 \mid x_{t}\right)\right) x_{t i} x_{t j}\right\}\right| \\
\leq & L_{0} T^{-1} \sum_{t=1}^{T} E\left(\left|T^{-1 / 2} x_{t}^{\prime} \Delta\right| \times\left|x_{t i}\right| \times\left|x_{t j}\right|\right),
\end{aligned}
$$

where the inequality is obtained by the triangle inequality, the Jensen's inequality, the Lipschitz continuity and the fact that $0 \leq\left|\xi_{T, t}\right| \leq\left|T^{-1 / 2} x_{t}^{\prime} \Delta\right|$. By the moment condition in Assumption 1(ii), the last expression in (9) con-

verges to zero, which implies that $E\left\{T^{-1} \sum_{t=1}^{T} g\left(\xi_{T} \mid x_{t}\right) x_{t} x_{t}^{\prime}\right\} \rightarrow E\left\{g\left(0 \mid x_{t}\right) x_{t} x_{t}^{\prime}\right\}$. Hence, we have $E\left(M_{T}(\Delta)\right)-E\left(M_{T}(0)\right) \rightarrow-Q \Delta$ as $T \rightarrow \infty$. Then, we substitute $E\left(M_{T}(\Delta)\right)-E\left(M_{T}(0)\right)$ in (7) with its limit $-Q \Delta$ to obtain the conclusion of the lemma. QED.

Proof of Proposition 1. We first define $\hat{\Delta}_{0}=\sqrt{T}\left(\hat{\Pi}-\Pi_{0}\right) \gamma_{0}$. Then, $\hat{\Delta}_{0}=O_{p}(1)$ by assumption. Hence, by Lemma 1 , we have $M_{T}\left(\hat{\Delta}_{0}\right)=$ $M_{T}(0)-Q \hat{\Delta}_{0}+o_{p}(1)$. The first term is given by

$$
M_{T}(0)=T^{-1 / 2} \sum_{t=1}^{T} x_{t} \psi_{\sigma}\left(v_{t}\right),
$$

which converges in distribution to a normal random variable by the LindebergLevy CLT under Assumptions 1 and 2(ii). Since $M_{T}(0)=O_{p}(1)$ and 
$Q \hat{\Delta}_{0}=O_{p}(1)$, using Lemma 1 , we obtain

$$
M_{T}\left(\hat{\Delta}_{0}\right)=O_{p}(1)
$$

Next, we define

$$
\hat{\Delta}_{1}(\delta)=H(\hat{\Pi}) \delta+\hat{\Delta}_{0}
$$

where $\delta \in R^{G+K_{1}}$. It is easily shown that Lemma 1 implies

$$
\sup _{\|\delta\| \leq L_{1}}\left\|M_{T}\left(\hat{\Delta}_{1}(\delta)\right)-M_{T}(0)+Q \hat{\Delta}_{1}(\delta)\right\|=o_{p}(1)
$$

for some finite $L_{1}>0$. We further define $\tilde{M}_{T}(\delta)=H(\hat{\Pi})^{\prime} M_{T}\left(\hat{\Delta}_{1}(\delta)\right)$ and $\|H(\hat{\Pi})\|^{2}=\operatorname{tr}\left(H(\hat{\Pi}) H(\hat{\Pi})^{\prime}\right)=O_{p}(1)$. By using the argument displayed between (A.7) and (A.8) in Powell (1983), it is shown that (10) and (12) together imply that

$$
\sup _{\|\delta\| \leq L_{1}}\left\|\tilde{M}_{T}(\delta)-H\left(\Pi_{0}\right)^{\prime} M_{T}\left(\hat{\Delta}_{0}\right)+Q_{z z} \delta\right\|=o_{p}(1)
$$

where $Q_{z z}=H\left(\Pi_{0}\right)^{\prime} Q H\left(\Pi_{0}\right)$. The next step of the proof is to show $\hat{\delta}=$ $T^{1 / 2}(\hat{\alpha}-\alpha)=O_{p}(1)$ in order to plug into (13). For this, we use Lemma A.4. in Koenker and Zhao (1996), which can be applied under the following conditions: (i) $-\delta^{\prime} \tilde{M}_{T}(\lambda \delta) \geq-\delta^{\prime} \tilde{M}_{T}(\delta)$ for $\lambda \geq 1$, (ii) $\left\|H\left(\Pi_{0}\right)^{\prime} M_{T}\left(\hat{\Delta}_{0}\right)\right\|=O_{p}(1)$, (iii) $\tilde{M}_{T}(\hat{\delta})=o_{p}(1)$, where $\hat{\delta}=T^{1 / 2}\left(\hat{\alpha}-\alpha_{0}\right)$, (iv) $Q_{z z}$ is positive-definite. With these conditions, Lemma A.4 in Koenker and Zhao will deliver the desired results: $\hat{\delta}=T^{1 / 2}(\hat{\alpha}-\alpha)=O_{p}(1)^{4}$. First, we note that the following function $h(\lambda)$ is convex in $\lambda: h(\lambda)=\sum_{t=1}^{T} \rho_{\sigma}\left(v_{t}-T^{-1 / 2} x_{t}^{\prime} H(\hat{\Pi}) \delta \lambda-\right.$ $\left.T^{-1 / 2} x_{t}^{\prime} \hat{\Delta}_{0}\right)$.Since $-\delta^{\prime} \tilde{M}_{T}(\lambda \delta)$ is the gradient of the above convex function, it is non-decreasing in $\lambda$. Hence, condition (i) is satisfied. The result in (10) implies condition (ii). To prove (iii), we note that

$$
T^{1 / 2} \tilde{M}_{T}(\hat{\delta})=\left[\left.\frac{\partial S_{T}}{\partial \alpha}\right|_{\alpha=\hat{\alpha}}\right]_{-}+o_{p}(1)
$$

where $\left[\left.\frac{\partial S_{T}}{\partial \alpha}\right|_{\alpha=\hat{\alpha}}\right]_{-}$is the vector of left-hand-side partial derivatives of the objective function $S_{T}$ in (5) evaluated at the solution $\hat{\alpha}$. Hence, $\left[\left.\frac{\partial S_{T}}{\partial \alpha}\right|_{\alpha=\hat{\alpha}}\right]_{-}=o_{p}(1)$.

The difference of order $o_{p}(1)$ in (14) comes from the fact that the scaled estimator $\hat{\sigma}$ is used on the right-hand-side (i.e. in the objective function $S_{T}$ ) while the true value $\sigma$ is used on the left-hand-side (i.e. in the definition of $\left.\tilde{M}_{T}(\hat{\delta})\right)$. Since $\hat{\sigma} \stackrel{p}{\rightarrow} \sigma$, the difference converges to zero in probability as $T$

\footnotetext{
${ }^{4}$ Once we show $\hat{\delta}=O_{p}(1)$, the consistency of $\hat{\alpha}$ for $\alpha_{0}$ follows by a by-product.
} 
goes to infinity. Hence, $T^{1 / 2} \tilde{M}_{T}(\hat{\delta})=o_{p}(1)$ and condition (iii) is satisfied. The final condition (iv) is ensured 2(iii) and by the identification condition in Assumption 2(iv). Therefore, we have $T^{1 / 2}\left(\hat{\alpha}-\alpha_{0}\right)=O_{p}(1)$. This result combined with (13) results in

$$
T^{1 / 2}\left(\hat{\alpha}-\alpha_{0}\right)=Q_{z z}^{-1} H\left(\Pi_{0}\right)^{\prime} M_{T}\left(\hat{\Delta}_{0}\right)+o_{p}(1)
$$

which delivers the desired result in the proposition by using the above decomposition of $M_{T}\left(\hat{\Delta}_{0}\right)$ provided by Lemma 1.:

$$
\begin{aligned}
T^{1 / 2}\left(\hat{\alpha}-\alpha_{0}\right)= & Q_{z z}^{-1} H\left(\Pi_{0}\right)^{\prime}\left\{T^{-1 / 2} \sum_{t=1}^{T} x_{t} \psi_{\sigma}\left(v_{t}\right)\right. \\
& \left.-Q T^{1 / 2}\left(\hat{\Pi}-\Pi_{0}\right) \gamma_{0}\right\}+o_{p}(1),
\end{aligned}
$$

which completes the proof. ${ }^{5}$ QED.

Proof of Proposition 2. In a fashion similar to Proposition 1 the conditions in Assumption 3 together with Assumptions 1 and 2(iv) are sufficient to show that the first stage Huber estimator $\hat{\Pi}_{j}$ has the following representation: $T^{1 / 2}\left(\hat{\Pi}_{j}-\Pi_{0 j}\right)=Q_{j}^{-1} T^{-1 / 2}$

$\sum_{t=1}^{T} x_{t} \psi_{\sigma_{j}}\left(V_{j t}\right)+o_{p}(1)$. Hence, $T^{1 / 2}\left(\hat{\Pi}-\Pi_{0}\right) \gamma_{0}=\sum_{j=1}^{G} Q_{j}^{-1} T^{-1 / 2}$

$\sum_{t=1}^{T} x_{t} \psi_{\sigma_{j}}\left(V_{j t}\right) \gamma_{0 j}+o_{p}(1)$, which is in turn plugged into (15) to deliver the following result:

$$
\begin{aligned}
T^{1 / 2}\left(\hat{\alpha}-\alpha_{0}\right)= & T^{-1 / 2} \sum_{t=1}^{T} Q_{z z}^{-1} H\left(\Pi_{0}\right)^{\prime} x_{t} \psi_{\sigma}\left(v_{t}\right) \\
& -T^{-1 / 2} \sum_{t=1}^{T} Q_{z z}^{-1} H\left(\Pi_{0}\right)^{\prime} Q Q_{1}^{-1} x_{t} \psi_{\sigma_{1}}\left(V_{1 t}\right) \gamma_{01} \\
& \ldots \\
& -T^{-1 / 2} \sum_{t=1}^{T} Q_{z z}^{-1} H\left(\Pi_{0}\right)^{\prime} Q Q_{G}^{-1} x_{t} \psi_{\sigma_{G}}\left(V_{G t}\right) \gamma_{0 G}+o_{p}(1) \\
= & D T^{-1 / 2} \sum_{t=1}^{T} Z_{t}+o_{p}(1)
\end{aligned}
$$

where $D=Q_{z z}^{-1} H\left(\Pi_{0}\right)^{\prime}\left[I,-Q Q_{1}^{-1} \gamma_{01}, \ldots,-Q Q_{G}^{-1} \gamma_{0 G}\right], Z_{t}=W_{t} \otimes x_{t}$, and $W_{t}=\left[\psi_{\sigma}\left(v_{t}\right), \psi_{\sigma_{1}}\left(V_{1 t}\right), \ldots, \psi_{\sigma_{G}}\left(V_{G t}\right)\right]^{\prime}$. QED.

\footnotetext{
${ }^{5}$ Since the objective function in the second stage is convex, an alternative proof to the presented one might have been obtained using the insightful approach of Hjort and Pollard (1993). We use Koenker and Zhao (1996) instead of Hjort and Pollard (1993) because we are more familiar with Koenker and Zhao (1996) on the one hand, and on the other hand because a parallel can thus be drawn with the proof in Kim and Muller (2004) for the two-stage quantile regression.
} 
Proof of Proposition 3. We first derive the distributional limit of $T^{-1 / 2} \sum_{t=1}^{T} Z_{t}$. Assumption 1(i) implies that $Z_{t}$ is independent and identically distributed. Using Assumptions 2(ii), 3(ii) and the law of iterated expectation, one can show that $E\left(Z_{t}\right)=0$. Finally, we note that $\operatorname{var}\left(Z_{t}\right)=E\left(W_{t} W_{t}^{\prime} \otimes x_{t} x_{t}^{\prime}\right)$ and all the elements in $W_{t} W_{t}^{\prime}$ are bounded by a constant. Hence, Assumption 1(ii) is sufficient to confirm that $\operatorname{var}\left(Z_{t}\right)$ is bounded. Therefore, we now can apply the Lindeberg-Levy's CLT to obtain $T^{-1 / 2} \sum_{t=1}^{T} Z_{t} \rightarrow N(0, \Omega)$. The conclusion of the proposition follows. QED. 


\section{References}

[1] Abadie, A., J. Angrist and G. Imbens, 2002, "Instrumental Variables Estimates of the Effect of Subsidized Training on the Quantiles of Trainee Earnings," Econometrica, 70, 91-117.

[2] Amemiya, T., 1982. Two stage least absolute deviations estimators. Econometrica 50, 689-711.

[3] Amemiya, T., 1985. The nonlinear two-stage least-squares estimation. Journal of Econometrics 2, 105-110.

[4] Andrews, D.W.K., 1994. Empirical process methods in econometrics. In: Engle, R.F., McFadden, D.L. (Eds.), Handbook of Econometrics, IV. New York, North-Holland, 2247-2294.

[5] Bickel, P.J., 1975. One-step Huber estimates in the linear model. Journal of the American Statistical Association 70, 428-433.

[6] Carroll, R.J., Ruppert, D., 1982. Robust estimation in heteroscedastic linear models. Annals of Statistics 10, 429-441.

[7] Chernozhukov, V. and C. Hansen, 2001, "An IV Model of Quantile Treatment Effects," Working Paper 02-06, Massachussets Institute of Technology, Department of Economics, December.

[8] Duncan. G.M., 1987. A simplified approach to M-estimation with application to two-stage estimators. Journal of Econometrics 34, 373-389.

[9] Flavin, M., 1999. Robust estimation of the joint consumption/asset demand decision. NBER Working Paper No. 7011.

[10] Hahn, J., 1995, "Bootstrapping Quantile Regression Estimators," Econometric Theory, 11, 105-121.

[11] Heckman, J.J., 1978. Dummy endogenous variables in a simultaneous equation system. Econometrica 46, 931-960.

[12] Hjort, N.L., Pollard, D., 1993. Asymptotics for minimisers of convex processes. Preprint, Department of Statistics, Yale University.

[13] Huber, P.J., 1964. Robust estimation of a location parameter. Annals of Mathematical Statistics 35, 73-101.

[14] Huber, P.J., 1981. Robust Statistics. New York, Wiley. 
[15] Kim, T.-H., Muller, C., 2004. Two-stage quantile regression when the first-stage is a quantile regression. Econometrics Journal 7, 218-231.

[16] Koenker, R., Portnoy, S., 1990. M-estimation of multivariate regressions. Journal of American Statistical Association 85, 1060-1068.

[17] Koenker, R., Zhao, Q., 1996. Conditional quantile estimation and inference for ARCH models. Econometric Theory 12, 793-813.

[18] Krasker, W.S., Welsch, R.E., 1985. Resistant estimation for simultaneous-equations models using weighted instrumental variables. Econometrica 53, 1475-1488.

[19] Krasker, W.S., 1986. Two-stage bounded-influence estimators for simultaneous-equations models. Journal of Business \& Economic Statistics $4,437-444$.

[20] Krishnakumar, J., Ronchetti, E., 1997. Robust estimators for simultaneous equations models. Journal of Econometrics 78, 295-314.

[21] Malinvaud, E., 1970. The consistency of nonlinear regressions. Annals of Mathematical Statistics 41, 956-969.

[22] Maronna, R.A., Yohai, V.J., 1997. Robust estimation in simultaneous equations models. Journal of Statistical Planning and Inference 57, 233244.

[23] Newey, W.K., 1985. Semiparametric estimation of limited dependent variables models with endogenous explanatory variables. Annales de l'INSEE 59/60, 219-235.

[24] Newey, W.K., 1989. A method of moments interpretation of sequential estimators. Economics Letters 14, 201-206.

[25] Newey, W.K., 1994. The asymptotic variance of semiparametric estimators. Econometrica 62, 1349-1382.

[26] Pagan, A.R., 1986. Two stage and related estimators and their applications. Review of Economic Studies 57, 517-538.

[27] Powell, J., 1983. The asymptotic normality of two-stage least absolute deviations estimators. Econometrica 51, 1569-1575. 
Table 1. Simulation Means and Standard Deviations of the Deviations from the True Value with: $\mathrm{N}(0,1)$

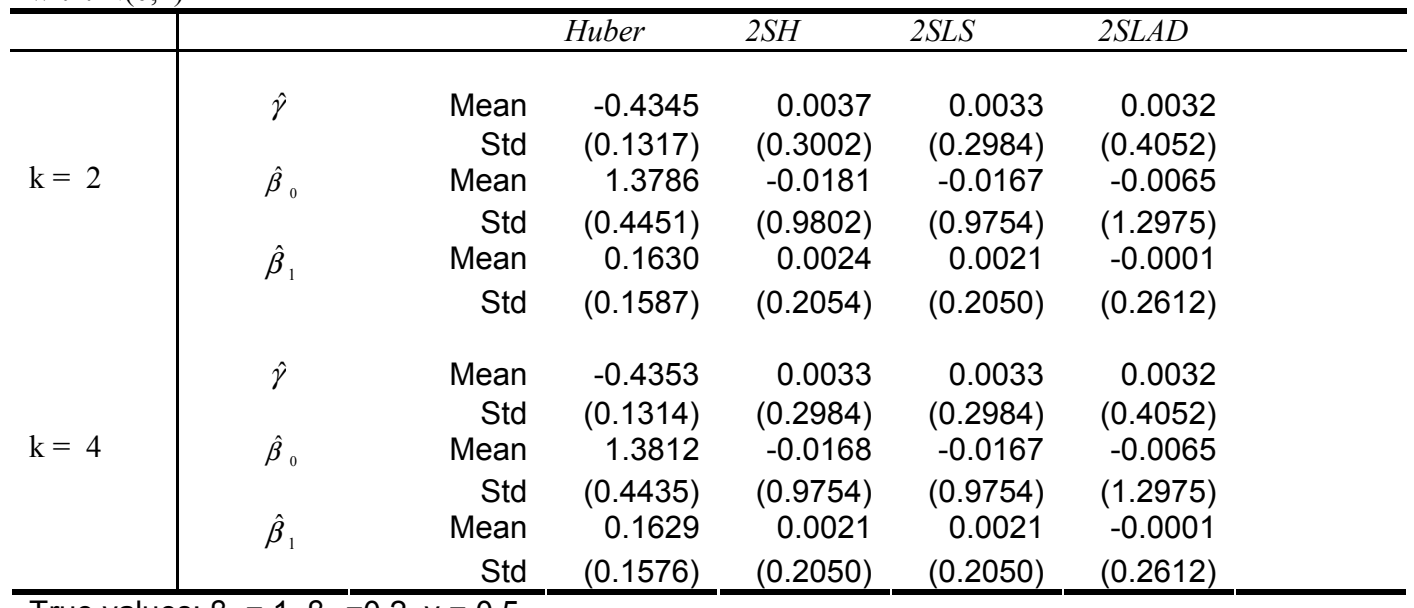

True values: $\beta_{0}=1, \beta_{1}=0.2, \gamma=0.5$

Table 2. Simulation Means and Standard Deviations of the Deviations from the True Value with: $\mathrm{t}(3)$

\begin{tabular}{|c|c|c|c|c|c|c|}
\hline & & & Huber & $2 \mathrm{SH}$ & $2 S L S$ & $2 S L A D$ \\
\hline \multirow{6}{*}{$\mathrm{k}=2$} & $\hat{\gamma}$ & Mean & -0.4987 & -0.0076 & -0.0282 & 0.0043 \\
\hline & \multirow{2}{*}{$\hat{\beta}_{0}$} & Std & $(0.1194)$ & $(0.3957)$ & $(0.6176)$ & $(0.4880)$ \\
\hline & & Mean & 1.5965 & 0.0382 & 0.1045 & -0.0022 \\
\hline & \multirow{3}{*}{$\hat{\beta}_{1}$} & Std & $(0.4359)$ & (1.2683) & (1.9703) & (1.5557) \\
\hline & & Mean & 0.1829 & -0.0041 & -0.0035 & -0.0043 \\
\hline & & Std & $(0.2031)$ & $(0.2827)$ & $(0.4051)$ & $(0.3132)$ \\
\hline \multirow{6}{*}{$\mathrm{k}=4$} & \multirow[t]{2}{*}{$\hat{\gamma}$} & Mean & -0.5129 & 0.0017 & -0.0282 & 0.0043 \\
\hline & & Std & $(0.1318)$ & $(0.5617)$ & $(0.6176)$ & $(0.4880)$ \\
\hline & \multirow[t]{2}{*}{$\hat{\beta}_{0}$} & Mean & 1.6456 & 0.0142 & 0.1045 & -0.0022 \\
\hline & & Std & $(0.4833)$ & $(1.7668)$ & (1.9703) & (1.5557) \\
\hline & \multirow[t]{2}{*}{$\hat{\beta}_{1}$} & Mean & 0.1855 & -0.0092 & -0.0035 & -0.0043 \\
\hline & & Std & $(0.2196)$ & $(0.3380)$ & $(0.4051)$ & $(0.3132)$ \\
\hline
\end{tabular}

